\title{
Supporting Physicians and Practice Teams in Efforts to Address the Opioid Epidemic
}

\author{
Lisa M. Letourneau, $M D, M P H^{1}$ \\ Jolie Ritzo, $\mathrm{MPH}, \mathrm{CHC}^{2}$ \\ Richard Shonk, $M D^{3}$ \\ Mark Eichler, $R P b^{4}$ \\ Stevi Sy, PharmD ${ }^{4}$ \\ 'Maine Department of Health \& Human \\ Services, Augusta, Maine \\ ${ }^{2}$ Network for Regional Healthcare Improve- \\ ment, South Portland, Maine \\ ${ }^{3}$ The Health Collaborative, Cincinnati, Ohio \\ ${ }^{4}$ Mountain-Pacific Quality Health, Casper, \\ Wyoming
}

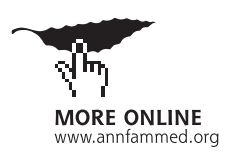

Conflicts of interest: authors report none.

\section{CORRESPONDING AUTHOR}

Lisa M. Letourneau, MD, MPH

Maine Department of Health \& Human

Services

109 Capitol St

11 State House Station

Augusta, ME 04333

letourneau.lisa@gmail.com

\begin{abstract}
Primary care physicians and practice teams increasingly recognize the need to take a role in addressing the growing epidemic of opioid use disorder (OUD) and opioid-related drug overdose deaths, but face considerable challenges in doing so. Through its work supporting practice transformation efforts, sharing innovations, and connecting key sectors within communities, the Network for Regional Healthcare Improvement and several of its member regional health improvement collaboratives have identified innovative ways to support physicians and practice teams in transforming practice in ways that address a spectrum of issues related to opioid use. These strategies include efforts to prevent, identify, and treat opioid addiction, including reducing inappropriate prescribing; improving opioid prescribing safety; compassionately tapering chronic and/or high-dose opioid regimens; appropriately screening for and identifying OUD; initiating treatment with evidence-based medications for OUD within practice settings; and prescribing life-saving naloxone to reverse opioid overdose. By outlining specific initiatives and practice transformation efforts that several regional health improvement collaboratives across the country have implemented to support clinicians and their teams, this article offers examples of how clinicians can get support from collaboratives in their region to implement practice improvement efforts to advance this critically important work.
\end{abstract}

Ann Fam Med 2019;17:S77-S81. https://doi.org/10.1370/afm.2408.

\section{INTRODUCTION}

$\mathrm{T}$ he related epidemics of substance use disorders (SUDs), opioid use disorder (OUD), and drug overdose deaths have continued to grow in the United States over the past several years. Despite increasing attention and widening action at state and national levels, drug overdose deaths have more than tripled in the last 7 years. ${ }^{1}$ Age-adjusted death rates have increased from 6.1 per 100,000 population in 1999 to 21.7 in 2017, with even higher rates in many states. This situation constitutes a national public health crisis, with drug overdose deaths now ranked as the third highest cause of death in the United States, contributing to a rise in the mortality rate of working-age adults in rural populations and exacting a toll on many individuals, families, and communities. ${ }^{2,3}$

With a steady rise in the prevalence of SUDs and OUD, primary care physicians (PCPs) and practice teams across the country are seeing an increasing number of patients in need of treatment for these disabling and all-too-frequently deadly conditions, but often struggle to identify roles they can take to address this crisis. At a national level, physicians have been slow to adopt recommendations to offer medication for addiction treatment (MAT) such as methadone and buprenorphine, which have been demonstrated to improve disease remission and reduce drug-related mortality. ${ }^{4}$ PCPs and practice teams face barriers when addressing or offering SUD and OUD treatment, including time constraints; resistance from practice partners; lack of available mental health and psychosocial supports; lack of addiction specialty support for referral or collaboration; and concerns about diversion or misuse of MAT. ${ }^{5}$ Despite national guidelines 
and a range of efforts to address opioid prescribing, ${ }^{6}$ rates of illicit drug use and OUD prevalence have continued to increase steadily, ${ }^{7}$ with sizable treatment gaps remaining ${ }^{8}$ and drug overdose death rates continuing to rise in most states. ${ }^{9}$ Clearly, more work is needed.

Stigma is an overarching issue affecting all aspects of SUDs and OUD diagnosis, treatment, and recovery. Studies show many clinicians, policymakers, and members of the public hold beliefs that may interfere with effective diagnosis and treatment, including implicit or explicit beliefs that addiction is a moral failure, not a chronic condition, doubts about the effectiveness of treatment and MAT ("substituting one drug for another"); and the belief that using naloxone increases risk-taking behavior. ${ }^{10,11}$ In addition to practice changes, efforts must be made to help clinicians and practice teams identify and address stigma.

\section{ROLE OF NETWORK FOR REGIONAL HEALTHCARE IMPROVEMENT}

As a national network of more than 30 nonprofit, multistakeholder regional health improvement collaboratives (RHICs) working to transform health and health care, the Network for Regional Healthcare Improvement (NRHI) and its members bring together a broad spectrum of clinicians, payers (health plans), health care purchasers (employers), and other community members to address health issues. RHICs are independent, nonprofit organizations that collectively provide services in 32 states, serving as neutral conveners and demonstrating effectiveness in improving health, quality, and affordability. These collaboratives variably serve as Centers for Medicare \& Medicaid Services (CMS)contracted Practice Transformation Networks, Support and Alignment Networks, and Quality Innovation Network-Quality Improvement Organizations, as well as health information technology organizations, Regional Extension Centers, and health information exchanges. Because of their multistakeholder nature, RHICs offer ready-made coalitions that can innovate, implement, and spread practice improvement strategies.

\section{NRHI MEMBERS AND REGIONAL APPROACHES}

The NRHI and several of its RHICs have identified innovative ways to support primary care practices in addressing SUDs and OUD. Recognizing the range of its members' opioid-related efforts, NRHI convened an Opioid Affinity Group in January 2018 to share best practices and work collaboratively. Through this group, members have gained better understanding of opportunities to address the opioid epidemic and participate in policy initiatives in their communities, including the role of RHICs as regional conveners. Several RHICs have implemented a range of efforts to help physicians and practice teams address the SUDs and OUD epidemic, as illustrated by 3 organizations: Quality Counts, The Health Collaborative, and Mountain-Pacific Quality Health (Mountain-Pacific). We describe these organizations and their activities below, and summarize them in the Supplemental Appendix (available at http://www.AnnFamMed.org/ content/17/Suppl_1/S77/suppl/DC1/).

\section{Quality Counts}

Since 2014, Quality Counts has been active in Maine, New Hampshire, and Vermont, leading several improvement efforts that help PCPs and practice teams address OUD, including efforts through its role as the Northern New England Practice Transformation Network.

\section{Coleading Caring for ME}

Quality Counts has partnered with the Maine Medical Association to lead Caring for ME, a statewide effort of education, support, and advocacy to engage clinicians and communities in building a stronger, compassionate response to the opioid epidemic. In this role, Quality Counts has hosted monthly meetings of clinician and community leaders statewide to share best practices; curated OUD educational resources and clinical tools; and sponsored monthly educational webinars.

\section{Promoting Stigma Reduction}

Quality Counts offers education and networking to help communities and clinicians understand SUDs and OUD as chronic conditions that benefit from evidence-based treatment, including in-person educational sessions and community forums, and tools for using first-person, nonstigmatizing language when treating patients.

Offering a Chronic Pain Collaborative and Playbook Quality Counts conducted 2 rounds of a Chronic Pain Collaborative during 2014-2016 to provide structured support and learning to teams from more than 20 primary care practices and thereby help them implement better systems for opioid prescribing. This undertaking led to the development of the Chronic Pain \& Controlled Medications Playbook, a toolkit that provides practical tools for approaching chronic pain management and prescribing of controlled medications. ${ }^{12}$

Supporting State Regulation/Legislation Compliance and Compassionate Opioid Tapering

In 2016, Maine passed legislation placing limits on opioid prescribing duration and amount. Quality Counts 
offered educational support and resources to help clinicians comply with this new law while maintaining a compassionate approach to patients receiving opioid therapy. Under the new law and with Quality Counts and partner support, Maine saw a 25\% decrease in opioid prescribing from 2016 to 2017, the largest decrease in the country that year. ${ }^{13}$

\section{Providing Online Learning Modules}

Quality Counts developed 8 online modules on chronic pain management; safe opioid prescribing to support compliance with Maine's law regulating opioid prescribing; and compassionate tapering. ${ }^{14}$ These 1-hour modules offer continuing medical education credit, are available to all clinicians, and were accessed through more than 1,700 encounters by 1,482 clinicians in 2017.

\section{Applying Project ECHO for Opioid-Related Topics} Quality Counts is a replication site for Project ECHO (Extension for Community Healthcare Outcomes), an evidence-based program designed to spread specialty knowledge and improve care for complex conditions..$^{15}$ Since 2016, Quality Counts has led the Northern New England ECHO program with more than 50 practices and 140 clinicians and team members, offering programs on a range of opioid-related issues including compassionate tapering of opioids; improvement of SUDs and OUD care in the perinatal period; and MAT.

\section{Convening Community Partners to Decrease Overdose Death}

Quality Counts is also using the ECHO model to convene 10 community coalitions to strengthen their capacity to prevent drug overdose deaths. Specifically, it seeks to engage clinicians to focus on key strategies, including collecting and monitoring data on overdose deaths; promoting rapid-access, low-barrier OUD treatment; promoting widespread availability of naloxone $_{i}$ and building links to recovery supports.

\section{The Health Collaborative}

The Health Collaborative brings together community, health care, health plan, and government stakeholders to address health issues in southwest Ohio and northern Kentucky. It operates one of the most longstanding health information exchange services in the country and leads the largest of the 18 regional Comprehensive Primary Care Plus $(\mathrm{CPC}+)$ demonstration projects. ${ }^{16}$ This work has allowed The Health Collaborative to leverage several of its existing programs to address the opioid epidemic within the greater Cincinnati area, a region hard hit by this crisis.

\section{Increasing MAT Capacity}

Using its CPC+ presence in Ohio, The Health Collaborative has integrated SUDs and OUD treatment education into its CPC+ educational events and provided training to improve capacity for offering MAT in primary care. The collaborative has also highlighted $\mathrm{CPC}+$ practices that provide MAT services, sharing best practices, resources, and models to enable OUD treatment, including efforts to engage many small, independent practices.

\section{Promoting SBIRT Training and Adoption}

The Health Collaborative worked with 6 hospital-based health systems to implement screening, brief intervention, and referral for treatment (SBIRT) in hospital emergency departments and inpatient units, especially maternity units. This unified approach to SUD screening as routine practice helped reduce associated stigma.

\section{Implementing an Emergency Department Treatment Protocol \\ Working through the Hamilton County Commis- sioner's Task Force, The Health Collaborative served as liaison with hospital leadership in communicating and integrating responses from emergency medical service professionals, first responders, law enforcement, and other community agencies to develop a standard, broadly accepted emergency department treatment protocol for SUDs and OUD.}

\section{Promoting the Community Score Card}

The Health Collaborative promoted to its stakeholders a Community Score Card developed by the Health Commissioner's Task Force that reflects SUDs and OUD key issues. This tool includes daily measurement of emergency department visits for OUD, overdose occurrences, emergency medical service runs, and a "heat map" showing the origin neighborhoods for drug overdoses. The Community Score Card has kept the crisis in front of the community, provided guidance to directing resources, and demonstrated progress on key measures.

\section{Conducting Primary Care Education and Training on Prescribing Patterns}

As Ohio state law changed to provide greater control over opioid prescribing patterns, The Health Collaborative used its links with clinicians to disseminate consistent messaging to physicians and allied health professionals on safer opioid-prescribing practices.

\section{Mountain-Pacific Quality Health}

Mountain-Pacific's broad geographic coverage spans Montana, Wyoming, Alaska, Hawaii, the Pacific Territories of Guam and American Samoa, and the Com- 
monwealth of Northern Mariana Islands. It serves as 1 of the 14 CMS Quality Innovation Network-Quality Improvement Organizations that provide quality improvement services to clinicians across its region, and it provides utilization review and pharmacy administrative services for the Montana Medicaid program. Through these contracts, Mountain-Pacific helps physicians and practice teams address the opioid epidemic

\section{Conducting Medicaid Drug Utilization \\ Review Activities}

Mountain-Pacific has led a number of initiatives aimed at improving the safety of opioid prescribing for Medicaid members.

\section{Supporting Safe Prescribing Practices and} Implementing Prescribing Limits

Mountain-Pacific provides education to pharmacy and medical professionals on safer opioid prescribing through newsletters and academic detailing. Education is centered on naloxone rescue therapy; opioid hyperalgesia; risks of opioid and benzodiazepine concurrent prescribing; and use of codeine-containing products in pediatric patients. Using Medicaid's morphine milligram equivalents limits, Mountain-Pacific implemented a program to limit prescribing of high-dose opioids for Medicaid recipients and developed an educational program for patients receiving opioids long term.

\section{Undertaking Data-Driven Interventions for Clinicians} Mountain-Pacific implemented data-driven interventions for clinicians, including programs to limit opioid prescribing at levels exceeding recommended morphine milligram equivalent limits; to identify patients at risk for opioid overdose who could benefit from naloxone rescue therapy; and to identify patients at increased risk for overdose from concurrent use of benzodiazepines and opioids.

\section{Conducting Medicare Activities}

Additionally, Mountain-Pacific has developed a range of activities with both clinicians and patients to improve the safety of opioid prescribing for Medicare beneficiaries.

\section{Offering Clinician Education}

Mountain-Pacific quality improvement staff provide multimodal education to clinicians on safe opioid prescribing; risks of adverse events from opioids, including overdose ; and the role of naloxone in opioid risk reduction. The organization hosts regional learning events that provide education, tools, and resources on reducing concurrent opioid and benzodiazepine use, encouraging adoption of Centers for Disease Control and
Prevention opioid-prescribing guidelines, and enhancing access to naloxone for patients and bystanders.

\section{Providing Beneficiary Education.}

Mountain-Pacific has also used several platforms to engage Medicare beneficiaries. Through social media, they have targeted beneficiaries in key demographic groups to provide education on medication safety and on signs and symptoms of adverse drug events. Campaign topics include medication take-back resources, safe medication storage, and use of naloxone.

\section{DISCUSSION}

Given the complexity and continued growth of the opioid epidemic in most states, physicians alone cannot bring about the solution but must be part of it, particularly given their critical role in improving prescribing safety and OUD diagnosis and treatment. Although clinical guidelines and national recommendations are helpful, evidence shows more is needed to implement practice changes, ${ }^{17,18}$ specifically, practical, real-time support that is available when clinicians need it and in a format in which they can best access it.

The NRHI and its member RHICs provide services to physician practices to address a range of issues related to the opioid epidemic. RHICs are well-respected conveners in their communities and uniquely positioned to assist PCPs and practice teams in a role not linked to proprietary interests, regulation, or oversight. They understand their communities' needs-especially in rural areas-and can help PCPs and practice teams link with other critical sectors, including state and local governments, law enforcement, and social service agencies. As leaders of successful practice transformation efforts, RHICs have the experience, trust, and relationships in their communities to work effectively with clinicians to address the fundamental, underlying issues of SUDs and OUD stigma and bias, and can leverage these relationships to innovate, implement, and sustain improvement efforts.

Many challenges remain, including insufficient numbers of clinicians who have completed the federally required training to prescribe medications for OUD, particularly in rural areas ${ }^{19}$; widely held perceptions that treating OUD in primary care settings is dangerous, complex, or both; stigma related to offering MAT services using opiate agonists; and a risky overemphasis on efforts to prescribe fewer opioids without a parallel commitment to increase the availability of OUD treatment for patients who may be addicted. ${ }^{20}$

Additionally, despite early efforts to move the US health care system from fee-for-service to value-based payments, most physicians still work within volume- 
based payment systems driven by productivity. It therefore remains challenging for physicians to take time out of practice to engage in activities that do not directly generate revenue.

Many RHICs have also found it challenging to secure resources to support SUDs and OUD intervention activities. Private foundations, which often fund other areas of practice change and innovation, generally look to federal and state entities to fund efforts to address the opioid epidemic. Although federal legislation has allocated considerable funds to address the opioid epidemic over the last several years, much of that funding is restricted to state entities, limiting access to organizations in states less willing to accept it.

In conclusion, PCPs and practice teams must be part of the solution to address the epidemic of opioid use, OUD, and overdose deaths. Despite considerable challenges in addressing these issues, clinicians are encouraged to identify RHICs in their community that can provide physicians and practice teams with muchneeded support to implement practice changes and community partnerships that are critical to addressing OUD. RHICs stand ready to work with physicians and communities to help them address the epidemic locally and, through collective efforts across the country, can work together to make a national impact.

To read or post commentaries in response to this article, see it online at http://www.AnnFamMed.org/content/17/Suppl_1/S77.

Key words: substance use disorders; opioids; practice transformation; organizational change; vulnerable populations; health policy; mental health; chronic illness; primary care; practice-based research

Submitted August 31, 2018; submitted, revised, January 9, 2019; accepted January 31, 2019.

Funding support: Support for time spent in preparation of this manuscript, as well as some of the work described, was provided in part by contracts with the Centers for Medicare $\&$ Medicaid Services (CMS) with Quality Counts, the Network for Regional Healthcare Improvement, The Health Collaborative, and Mountain-Pacific Quality Health. The organizations and individuals involved in providing support for this manuscript include the following: Quality Counts: Lisa M. Letourneau, MD, MPHwriting, editing, and submitting manuscript; The Health Collaborative: Richard Shonk, MD-writing and editing manuscript; Mountain-Pacific Health Quality: Marc Eichler, RPh, and Stevi Sy, PharmD-writing and editing manuscript; Network for Regional Health Improvement: Jolie Ritzo, MPH, CHC-writing and editing manuscript.

Supplementary Materials: Available at http://www.AnnFamMed. org/content/17/Suppl_1/S77/suppl/DC1/.

\section{References}

1. Hedegaard H, Minino A, Warner M. Drug Overdose Deaths in the United States: 1999-2017. NCHS Data Brief No. 329. Hyattsville, MD: National Center for Health Statistics; 2018. https://www.cdc.gov/ nchs/data/databriefs/db329-h.pdf. Published Nov 2018. Accessed Jan 5, 2019
2. Kochanek KD, Murphy SL, Xu J, Arias E. Mortality in the United States, 2016. NCHS Data Brief No. 293. Hyattsville, MD: National Center for Health Statistics; 2017. https://www.cdc.gov/nchs/data/ databriefs/db293.pdf. Published Dec 2017. Accessed Jan 5, 2018.

3. Rural America at a Glance: 2017 Edition. USDA Economic Information Bulletin 182. https://www.ers.usda.gov/webdocs/ publications/85740/eib-182.pdf?v=43054. Published Nov 2017. Accessed Aug 2, 2018.

4. Sordo L, Barrio G, Bravo MJ, et al. Mortality risk during and after opioid substitution treatment: systematic review and meta-analysis of cohort studies. BMJ. 2017;357:j1550.

5. Andrilla CHA, Coulthard C, Larson EH. Barriers rural physicians face prescribing buprenorphine for opioid use disorder. Ann Fam Med. 2017;15(4):359-362.

6. Centers for Disease Control and Prevention. CDC guideline for prescribing opioids for chronic pain. http://www.cdc.gov/drugoverdose/ prescribing/guideline.html. Updated Apr 17, 2019. Accessed Aug 3, 2018.

7. Mack KA, Jones CM, Ballesteros MF. Illicit drug use, illicit drug use disorders, and drug overdose deaths in metropolitan and nonmetropolitan areas - United States. MMWR Surveill Summ. 2017;66(19):1-12.

8. Jones CM, Campopiano M, Baldwin G, McCance-Katz E. National and state treatment need and capacity for opioid agonist medication-assisted treatment. Am J Public Health. 2015;105(8): e55-e63.

9. Hedegaard H, Minino A, Warner M. Drug Overdose Deaths in the United States: 1999-2016. NCHS Data Brief No. 294. https://www. cdc.gov/nchs/data/databriefs/db294.pdf. Published Dec 2017. Accessed Aug 3, 2018.

10. Wakeman SE, Pham-Kanter G, Donelan K. Attitudes, practices, and preparedness to care for patients with substance use disorder: Results from a survey of general internists. Subst Abus. 2016;37(4):635-641.

11. van Boekel LC, Brouwers EP, van Weeghel J, Garretsen HF. Stigma among health professionals towards patients with substance use disorders and its consequences for healthcare delivery: systematic review. Drug Alcohol Depend. 2013;131(1-2):23-35.

12. Maine Quality Counts. Chronic pain $\&$ controlled medication playbook. https://mainequalitycounts.org/what-we-do/populationhealth/chronic-pain-and-controlled-medication-playbook/. Accessed Aug 23, 2018.

13. Brantley K, Sloan C. Avalere report: opioid supply levels decline in most states. http://avalere.com/expertise/life-sciences/insights/ opioid-supply-levels-decline-in-most-states. Published Jun 19, 2018 Accessed Aug 3, 2018.

14. Maine Quality Counts Learning Lab. Caring for ME (safe opioid prescribing). https://qclearninglab.org/course-cat/caring-for-me/. Accessed Aug 23, 2018.

15. University of New Mexico. Project ECHO. https://echo.unm.edu/. Accessed May 20, 2019.

16. Centers for Medicare \& Medicaid Services. Comprehensive primary care plus. https://innovation.cms.gov/initiatives/ComprehensivePrimary-Care-Plus. Updated May 14, 2019. Accessed May 20, 2019.

17. Hurtado MP, Swift EK, Corrigan JM, eds. Crossing the Quality Chasm: A New Health System for the 21st Century. Washington, DC: National Academies Press; 2001.

18. Berwick DM.The science of improvement. JAMA. 2008; 299(10): 1182-1184.

19. Hancock C, Mennenga H, King N, et al. National Rural Health Association policy brief: treating the rural opioid epidemic. Accessed Aug 23, 2018. https://www.ruralhealthweb.org/NRHA/ media/Emerge_NRHA/Advocacy/Policy\%20documents/Treating-theRural-Opioid-Epidemic_Feb-2017_NRHA-Policy-Paper.pdf. Published Feb 2017.

20. Wakeman SE, Barnett ML. Primary care and the opioid-overdose crisis - buprenorphine myths and realities. N Engl J Med. 2018; $379(1): 1-4$. 